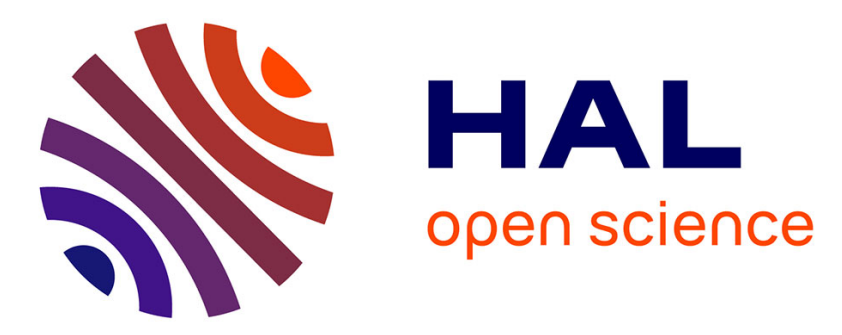

\title{
An Observer-Based Design for Cogging Forces Cancellation in Permanent Magnet Linear Motors
}

\author{
Jérémy Malaizé, Jean Lévine
}

\section{To cite this version:}

Jérémy Malaizé, Jean Lévine. An Observer-Based Design for Cogging Forces Cancellation in Permanent Magnet Linear Motors. 48th IEEE Conference on Decision and Control, Dec 2009, Shanghai, China. pp.6811, 10.1109/CDC.2009.5399831 . hal-00575205

\section{HAL Id: hal-00575205 \\ https: / hal-mines-paristech.archives-ouvertes.fr/hal-00575205}

Submitted on 9 Mar 2011

HAL is a multi-disciplinary open access archive for the deposit and dissemination of scientific research documents, whether they are published or not. The documents may come from teaching and research institutions in France or abroad, or from public or private research centers.
L'archive ouverte pluridisciplinaire HAL, est destinée au dépôt et à la diffusion de documents scientifiques de niveau recherche, publiés ou non, émanant des établissements d'enseignement et de recherche français ou étrangers, des laboratoires publics ou privés. 


\section{An Observer-Based Design for Cogging Forces Cancellation in Permanent Magnet Linear Motors}

\author{
Jérémy Malaizé (1) \\ (1) Institut Français du Pétrole \\ Rueil Malmaison, France \\ jeremy.malaizedifp. fr
}

\author{
Jean Lévine ${ }^{(2)}$ \\ (2) Mines ParisTech, Centre Automatique et Systèmes \\ Mathématiques et Systèmes \\ Fontainebleau, France \\ jean.levinedensmp.fr
}

\begin{abstract}
This paper adresses the high precision positioning issue of permanent magnet (PM) linear motors, in presence of spatially periodic forces, also known as cogging. Using an internal model representation of this perturbation, an observerbased controller only relying on position measurements is derived. The observation error is not autonomous, and the stability analysis of the resulting closed loop system is regarded as the stability of two interconnected systems. For the motor to quickly track a desired trajectory, while being robust to large magnitude cogging forces, a small-gain like theorem is derived and used to tune the gains of the control law in an explicit way. The experimental results obtained through this method are then showed and compared with those of a PID controller.
\end{abstract}

\section{INTRODUCTION}

This paper is concerned with motors used in high-end applications, such as wafer steppers central to the lithography process. These machines are expected to carry loads along point-to-point trajectories with stringent positioning accuracy requirements, usually ranging from a micron to a few dozens nanometers. Given this constraint, direct drive solutions are chosen to actuate these machines. They are actually capable of providing the sufficient precision levels and the required smoothness of motion by getting rid of undesirable phenomena such as hysteresis, backlash or mechanical play. In the following, we focus on the use of PM synchronous motors. They are preferred to DC motors for their longer life time, improved cleanliness of operation and better resistance to wear and tear.

Ironless motors feature two magnetic tracks, made up of permanent magnets, while ironcore motors feature only one magnetic track. The excess of ferromagnetic material used for this design allows to trap the magnetic field generated by this only track in the vicinity of the rotor windings. This costeffective design spares the use of expensive and eventually superfluous rare earths magnets and yields motors with better efficiency. However, this design generates perturbation forces, hereafter referred to as cogging that significantly affects their performances, in view of the desired accuracy requirements. Ironless motors, though much more expensive, turn out to be significantly less affected by cogging.

Cogging is due to the interaction of the magnetic field with the ferromagnetic material of the rotor. Given the periodic layouts of magnets and slots (see 1), cogging forces are shown to be spatially periodic perturbations, see [1], [2] or [3] for in depth reviews. Roughly speaking, even if not fed with electrical currents, these motors have a tendency to stabilize at specific positions so as to minimize magnetic energy.

This paper is concerned with getting PM synchronous motors to track reference trajectories with stringent accuracy specifications, related to the aforementioned applications, in spite of the cogging forces. These trajectories can be of any kind, meaning this paper is not limited to constant velocity motion along which these spatially periodic forces would readily come down to temporally periodic perturbations. Position measurements are assumed to be available, while the actual speed of the motor is not measured. The spatial periods of the cogging are known.

Great efforts have been made to minimize this, though natural, limitation of the ironcore motors from a technological point of view. The underlying idea consists in using the free design parameters, like the shapes of the magnets, the spacing in between slots, or the length of the rotor to analytically or numerically find out which configuration actually minimizes cogging forces. All these techniques generally lead to very complex designs, such as skewed magnets layouts, which, in the end, do not manage to completely get rid of cogging forces (see [4]). The same conclusion applies to ironless motors, but, for the motors considered hereafter, the main spatially perdiodic perturbations are due to unavoidable and slowly time-varying electrical current offsets rather than cogging. For the sake of simplicity, we shall also consider these forces as cogging forces.

Driving a production model motor with a PID controller yields satisfactory results for some applications, but, when stringent accuracy specifications are at stake, a dedicated controller is required to get rid of the oscillations around the desired trajectory induced by the residual cogging forces. It might be tempting to cancel these forces from a preliminary analytical or numerical analysis together with a feedforward compensation scheme, but modeling these perturbations turn out to be tough, see [4].

The previous analysis makes lean towards an online cogging compensation scheme. For repetitive tasks, the cogging forces affecting the motion during one run may be identified, and, directly compensated for during the next run. This iterative method, known as learning feedforward [5], can obviously cope with perturbations of arbitrary shape but takes 
several tries to yield satisfactory results.

Other techniques are dedicated to spatially periodic perturbations and rely on a spatial Fourier series expansion of cogging forces. Adaptive controllers may be designed to estimate both the magnitudes and phases of each of the Fourier series expansion components and achieve position tracking thanks to cogging compensation, see [6] and [7] for instance. Observer-based controllers can also be derived using an internal model by considering an extended system made up of the motor dynamics and the perturbations. In [8], this approach is implemented for mechanical pure sine perturbations but the extension to higher order harmonics does not seem straightforward.

The methods reported so far assume the velocity available, either by direct measurements or numerical differentiation of the position. For high precision positioning applications, assuming the position directly measured is rather sensible and common, but numerical computation of the velocity may generate undesirable noise affecting the overall system performances. In this paper, we propose an observer-based controller only relying on position measurements. Our solution steers the tracking error to zero by cancelling cogging forces defined by an arbitrary number of spatial periods, as well as compensating for Coulomb friction.

This paper is organized as follows. We first model the dynamics of the motors considered so far and give an analytical definition of the cogging forces (section II). An observer structure estimating the states of both the mechanics and the perturbation is proposed together with a controller fed with these estimated values to allow exact reference trajectory tracking despite cogging forces (section III). The corresponding gains have to be tuned to achieve perturbation rejection and for the motor to quickly rally the desired trajectory. The stability analysis (section IV) is mainly based on a small gain result, pointing out the robustness of the proposed observer-based controller with respect to the magnitude of the cogging forces. The performances of the method are then illustrated through experiments carried out on both ironless and ironcore motors (section V).

\section{PM SYNCHRONOUS MOTORS}

\section{A. Modeling}

Figure 1 depicts a two-phase ironcore motor (the modeling turns out to be the same for ironless motors and three-phase windings motors), where permanent magnets, periodically spread out along the stator, create a periodic magnetic field, with spatial period $P$, trapped in the rotor out of iron. The rotor carries windings and is free to move along the $x$-axis. The converse design with fixed windings and moving magnetic track might also be conceivable. It is rather classical to assume that a current controller stabilizes the dynamics of the electrical currents. They eventually turn out to be fast in comparison with the mechanical behavior of the motor. When using the field-oriented method to drive the currents while neglecting their dynamics, a force propels the motor ahead, see [9].

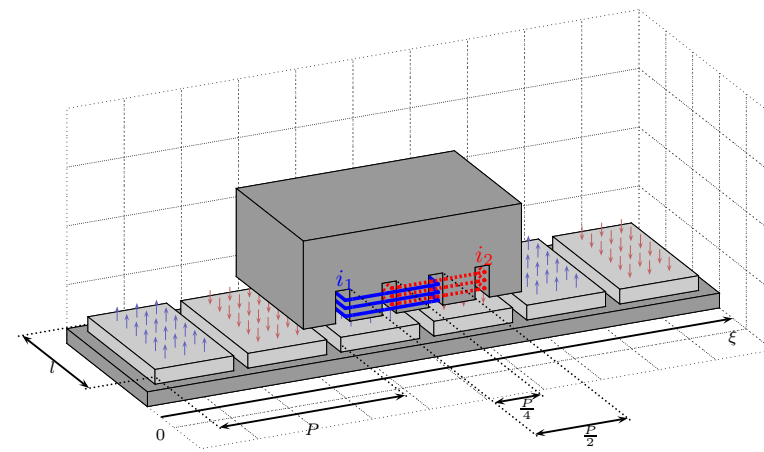

Fig. 1. Two-phase ironcore linear motor.

The remaining forces acting on the motor are due to cogging and friction. The dynamical behaviour of the motor with speed $\nu$ and measured position $y=x$ eventually reads:

$$
\begin{aligned}
\left(\begin{array}{c}
\dot{x} \\
\dot{\nu}
\end{array}\right) & =A\left(\begin{array}{l}
x \\
\nu
\end{array}\right)+B(u+\Lambda(x)-f \operatorname{sgn}(\nu)) \\
y & =C\left(\begin{array}{c}
x \\
\nu
\end{array}\right)
\end{aligned}
$$

where the notations are given by:

$$
A=\left(\begin{array}{cc}
0 & 1 \\
0 & -\mu
\end{array}\right), \quad B=\left(\begin{array}{l}
0 \\
1
\end{array}\right), \quad C=\left(\begin{array}{l}
1 \\
0
\end{array}\right)^{T} .
$$

The parameters $\mu$ and $f$ respectively model viscous and dry friction, $u$ is the controlled thrust and $\Lambda(x)$ models the action of cogging forces on the acceleration of the motor. Though friction is modeled by a differential inclusion, in our case, a simple constant parameter suffices to bring the desired performances to the derived observer-based controller. Moreover, for the considered applications, friction is definitely not the most significant limitation. However, the extension of our work to differential inclusions could be the scope of further research activities. From now on, the modeling (1), together with assuming friction to be a constant unknown parameter, will be used for either ironcore or ironless motors.

\section{B. Cogging forces}

According to [1], [2], the finite length of the rotor as well as the windings slots (see figure 1) are some of the design parameters inducing cogging forces. Each of them yields spatially periodic perturbation forces with a specifc period, which in the case of the finite length of the rotor turns out to be $\frac{P}{2}$. Current offsets generate cogging-like forces with the same spectral components as the back-emf.

Truncated Fourier series expansion is a rather well-suited tool to model the $x$-periodic function $\Lambda(x)$, made up of $N$ sine functions, as given by:

$$
\Lambda(x)=\lambda_{0}+\sum_{n=1}^{N} \lambda_{n} \sin \left(\frac{2 \pi}{P_{n}} x+\varphi_{n}\right),
$$

where the a priori unknown $\lambda_{n}$ 's and $\varphi_{n}$ 's bear magnitude and phase information of the $n$-th spectral component with known spatial period $P_{n}$. This approach copes with cogging 
forces made up of several fundamental spatial periods, and also with friction through $\lambda_{0}$.

Adaptive methods mentioned in the introduction mainly cope with the online estimation of the $\lambda_{n}$ 's and $\varphi_{n}$ 's in order to compensate for $\Lambda(x)$. In this paper, an observer-based method is proposed, and, to this end, $\Lambda(x)$ is expressed as the output of a dynamical system. For any $n, 1 \leq n \leq N$, let's note:

$\zeta_{n, 1}=\lambda_{n} \sin \left(\frac{2 \pi}{P_{n}} x+\varphi_{n}\right), \zeta_{n, 2}=\lambda_{n} \cos \left(\frac{2 \pi}{P_{n}} x+\varphi_{n}\right)$

and observe:

$$
\left(\begin{array}{l}
\dot{\zeta}_{n, 1} \\
\dot{\zeta}_{n, 2}
\end{array}\right)=\nu \frac{2 \pi}{P_{n}}\left(\begin{array}{cc}
0 & 1 \\
-1 & 0
\end{array}\right)\left(\begin{array}{l}
\zeta_{n, 1} \\
\zeta_{n, 2}
\end{array}\right):=\nu M_{n}\left(\begin{array}{l}
\zeta_{n, 1} \\
\zeta_{n, 2}
\end{array}\right)
$$

Moreover, let $\zeta_{0}=\lambda_{0}$, with $\dot{\zeta}_{0}=0$, and stacking it up together with the variables $\zeta_{n, 1}$ and $\zeta_{n, 2}$ in the vector $\zeta \in \mathbb{R}^{2 N+1}, \Lambda(x)$ turns out to be the output of a dynamical system, obtained using an internal model representation:

$$
\begin{aligned}
& \dot{\boldsymbol{\zeta}}=\nu\left(\begin{array}{c:cccc}
0 & \mathbf{0} & \ldots & \ldots & 0 \\
\hdashline \mathbf{0} & M_{1} & \mathbf{0} & \ldots & \mathbf{0} \\
\vdots & \mathbf{0} & \ddots & \ddots & \vdots \\
\vdots & \vdots & \ddots & \ddots & \mathbf{0} \\
\mathbf{0} & \mathbf{0} & \ldots & \mathbf{0} & M_{N}
\end{array}\right) \boldsymbol{\zeta}:=\nu \mathcal{M}_{N} \boldsymbol{\zeta} \\
& \Lambda(x)=\left(\begin{array}{c:cc:cc:c}
1 & 1 & 0 & \ldots & 1 & 0
\end{array}\right) \boldsymbol{\zeta}:=\mathcal{C}_{N} \boldsymbol{\zeta}
\end{aligned}
$$

where the matrices $M_{n}$ 's are defined by (3) and 0 stands for null matrices with appropriate dimensions.

\section{OBSERVER AND CONTROLLER DESIGN}

\section{A. Observer structure}

Let $\chi=\left(\begin{array}{lll}x & \nu & \zeta\end{array}\right)^{T}$ be the whole state of the plant, and, combining (1) together with (4):

$$
\begin{aligned}
\dot{\chi} & =\mathcal{A}(\nu) \chi+\mathcal{B}(u-f \operatorname{sign}(\nu)) \\
y & =\mathcal{C} \chi
\end{aligned}
$$

with notations as follows:

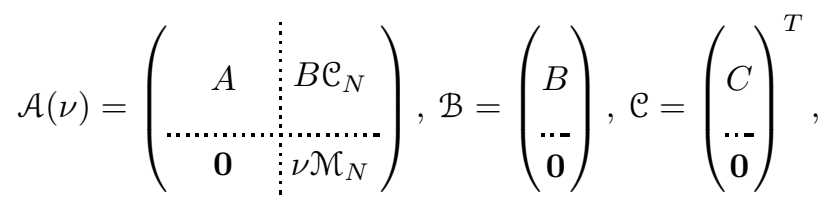

with 0 standing for null matrices with relevant dimensions. Let's note that, obviously, at standstill, the complete state $\boldsymbol{\zeta}$ is not observable. On top of that, as a perturbation, $\boldsymbol{\zeta}$ is not controllable but remains bounded according to its initial conditions.

The proposed observer architecture based on the only measurements of the position $x$ is the following:

$$
\dot{\hat{\chi}}=\mathcal{A}\left(\nu^{*}\right) \widehat{\chi}+\mathcal{B} u+\mathcal{K} \mathcal{C}(\chi-\widehat{\chi})
$$

To some extent, the proposed expression (6) is similar to a Luenberger observer in that it is clearly a copy of the dynamics (5) together with a correction term through gain
$\mathcal{K}$. However, note that dry friction is not allowed for in (6), but as a piecewise constant phenomenon, this term is merely estimated along with $\lambda_{0}$. Cautions have to be taken to properly reset the observer whenever the sign of the desired velocity $\nu^{*}$ changes. From now on, for convenience, $f$ is ommited in (5), but will still be taken into consideration for the simulation results.

The second point of interest is the use of the reference velocity $\nu^{*}$ in (6). The observer scheme (6) can be regarded as a kind of linearization of the perturbation, lower block in (5), around the reference trajectory $x^{*}$.

\section{B. Closed loop dynamics}

Our goal is to use this in parallel with a controller to achieve precise positioning. To this end, observation and positioning errors are denoted by:

$$
\begin{gathered}
\widetilde{x}=\widehat{x}-x, \quad \widetilde{\nu}=\widehat{\nu}-\nu, \quad \widetilde{\boldsymbol{\zeta}}=\widehat{\boldsymbol{\zeta}}-\boldsymbol{\zeta}, \\
\varepsilon_{x}=x-x^{*}, \quad \varepsilon_{\nu}=\nu-\nu^{*}
\end{gathered}
$$

and collected in the vectors $\chi_{o}=\left(\begin{array}{lll}\widetilde{x} & \widetilde{\nu} & \widetilde{\zeta}\end{array}\right)^{T}$ and $\chi_{c}=$ $\left(\begin{array}{ll}\varepsilon_{x} & \varepsilon_{\nu}\end{array}\right)^{T}$.

The following equation concerning the observation error is readily derived from (5) subtracted to (6):

$$
\dot{\chi}_{o}=\left(\mathcal{A}\left(\nu^{*}\right)-\mathcal{K} \mathcal{C}\right) \chi_{o}+B_{o} \varepsilon_{\nu} \mathcal{M}_{N} \boldsymbol{\zeta},
$$

where the matrix $B_{o}$ reads:

$$
B_{o}=\left(\begin{array}{c}
\mathbf{0} \\
\mathbf{0} \\
\hdashline \bar{I}_{2 N+1}
\end{array}\right)
$$

with 0 null matrices of relevant dimensions, and $I_{2 N+1}$ the identity matrix of dimenion $2 N+1$. Let us move on to the controller design from the previous estimated values. Provided the vector $\widehat{\zeta}$ converges to the actual vector $\zeta$, it is sensible to design the input $u$ to compensate for the perturbation $\Lambda=\mathcal{C}_{N} \zeta$. Additional state feedback through the gain matrix $\mathcal{L}=\left(\begin{array}{ll}L_{x} & L_{\nu}\end{array}\right)$ and feedforward terms are added to achieve exact trajectory tracking so that the controller eventually computes $u$ according to:

$$
u=-\mathcal{L}\left(\begin{array}{c}
x-x^{*} \\
\widehat{\nu}-\nu^{*}
\end{array}\right)-\mathcal{C}_{N} \widehat{\zeta}+\dot{\nu}^{*}+\mu \nu^{*}
$$

In the end, the full state space representation of (5) driven by the observer-based controller made up of (7) and (8) is given by:

$$
\begin{aligned}
& \dot{\chi}_{c}=(A-B \mathcal{L}) \chi_{c}-B C_{o} \chi_{o} \\
& \dot{\chi}_{o}=\left(\mathcal{A}\left(\nu^{*}\right)-\mathcal{K} \mathcal{C}\right) \chi_{o}+\varepsilon_{\nu} B_{o} \mathcal{M}_{N} \zeta
\end{aligned},
$$

whith $C_{o}=\left(\begin{array}{lll}0 & L_{\nu} & \mathrm{C}_{N}\end{array}\right)$. In (9), the evolution of the uncontrollable part $\zeta$ of (5) has been ommitted, as we are mainly concerned by the stability of the origin of (9) that is equivalent to both observation and positioning errors converging to zero.

Looking at (7), unlike classical Luenberger observers, the observation error is not completely autonomous, which results from the linearization of $\Lambda(x)$ around the reference 
trajectory. Fortunately, as the origin is still an equilibrium point of (9) despite the non-controllable part $\zeta$, it might not be hopeless to look for an appropriate tuning of $\mathcal{L}$ and $\mathcal{K}$ guaranteeing global asymptotic stability of the origin of the closed loop (9).

\section{Stability ANALYSis}

In (9), given the non-autonomous observation error, the observation $\mathcal{K}$ and positioning $\mathcal{L}$ gains tuning may not be decoupled. We shall assume that $\mathcal{L}$ is given by some a priori knowledge of the plant and $\mathcal{K}$ is derived using some results on the stability of interconnected systems. In doing so, we shall derive an interesting result concerning the robustness of the proposed observer structure versus the magnitude of the perturbation.

\section{A. Small-gain theorem for cogging cancellation}

Let us first have a fresh look at (9), and note that this equation can be viewed as the input-output interconnection of:

$$
\begin{aligned}
\dot{\chi}_{c} & =(A-B \mathcal{L}) \chi_{c}+B u_{c} \\
z_{c} & =\mathcal{M}_{N} \zeta C_{c} \chi_{c},
\end{aligned}
$$

with $C_{c}=\left(\begin{array}{ll}0 & 1\end{array}\right)$ and

$$
\begin{aligned}
\dot{\chi}_{o} & =\left(\mathcal{A}\left(\nu^{*}\right)-\mathcal{K} \mathcal{C}\right) \chi_{o}+B_{o} u_{o} \\
z_{o} & =C_{o} \chi_{o},
\end{aligned}
$$

with $u_{c}=-z_{o}$ and $u_{o}=z_{c}$. The following small-gain theorem, based on dissipativity, gives sufficient conditions for the observer-based cogging cancellation scheme based on the perturbation linearization to be globally asymptotically stable.

Theorem 1: [Small-gain theorem] Suppose the following properties are fulfilled:

(i) There exist a function $V_{c}: \mathbb{R}^{2} \rightarrow \mathbb{R}$, such that $V_{c}(0)=0$ and $\forall x \in \mathbb{R}^{2}-\{0\}, V_{c}(x)>0, a \mathcal{K}_{\infty}$ function $\alpha_{c}$ and a scalar $\gamma_{c}>0$ such that $\forall\left(\chi_{c}, u_{c}\right)$ :

$$
\dot{V}_{c}\left(\chi_{c}\right) \leq \gamma_{c}^{2} u_{c}^{T} u_{c}-\chi_{c}^{T} C_{c}^{T} C_{c} \chi_{c}-\alpha_{c}\left(\left\|\chi_{c}\right\|\right)
$$

(ii) There exist a function $V_{o}: \mathbb{R}^{2 N+3} \rightarrow \mathbb{R}$, such that $V_{o}(0)=0$ and $\forall x \in \mathbb{R}^{2 N+3}-\{0\}, V_{o}(x)>0, a \mathcal{K}_{\infty}$ function $\alpha_{o}$ and a scalar $\gamma_{o}>0$ such that $\forall\left(\chi_{o}, u_{o}\right)$ :

$$
\dot{V}_{o}\left(\chi_{o}\right) \leq \gamma_{o}^{2} u_{o}^{T} u_{o}-\chi_{o}^{T} C_{o}^{T} C_{o} \chi_{o}-\alpha_{o}\left(\left\|\chi_{o}\right\|\right)
$$

then the closed loop system is globally asymptotically stable if

$$
2 \pi \gamma_{c} \gamma_{o} \sqrt{\sum_{n=1}^{N}\left(\frac{\lambda_{n}}{P_{n}}\right)^{2}}<1
$$

where the $\lambda_{n}$ are the coefficients of the series expansion (2).

Proof: First, denote:

$$
\gamma_{N}=\sqrt{\sum_{n=1}^{N}\left(\frac{\lambda_{n}}{P_{n}}\right)^{2}}
$$

Let find a scalar $a>0$ for $V=V_{c}+a V_{o}$ to be a Lyapunov function of the whole system (9) with $u_{c}=-z_{o}$ and $u_{o}=$ $z_{c}$. Basic requirements concerning $V$ are readily checked, moreover, note that:

$$
z_{c}^{T} z_{c}=4 \pi^{2} \gamma_{N}^{2} \times \chi_{c}^{T} C_{c}^{T} C_{c} \chi_{c}
$$

and compute $\dot{V}\left(\chi_{c}, \chi_{o}\right)$ :

$$
\begin{aligned}
\dot{V}\left(\chi_{c}, \chi_{o}\right) \leq & \left(\gamma_{c}^{2}-a\right) z_{o}^{T} z_{o}+\left(a \gamma_{o}^{2}-\frac{1}{\left(2 \pi \gamma_{N}\right)^{2}}\right) z_{c}^{T} z_{c} \\
& -\alpha_{c}\left(\left\|\chi_{c}\right\|\right)-a \alpha_{o}\left(\left\|\chi_{o}\right\|\right)
\end{aligned}
$$

Suppose $\gamma_{c}^{2}<a<\frac{1}{4 \pi^{2} \gamma_{o}^{2} \gamma_{N}^{2}}$ then

$$
\dot{V}_{c}\left(\chi_{c}\right)+a \dot{V}_{o}\left(\chi_{o}\right) \leq-\alpha_{c}\left(\left\|\chi_{c}\right\|\right)-a \alpha_{o}\left(\left\|\chi_{o}\right\|\right)
$$

and global asymptotic stability is showed provided the sufficient condition $2 \pi \gamma_{c} \gamma_{o} \gamma_{N}<1$ is met.

Recalling that the observer is based on the linearization of the perturbation, this theorem states the intrinsic limitation of our approach. It also shows we are not completely helpless before the perturbation, for the gains $\mathcal{L}$ and $\mathcal{K}$ play a role in the values taken by $\gamma_{c}$ and $\gamma_{o}$.

\section{B. Gain tuning issues}

As stated by the following theorem, quadratic Lyapunov functions turn out to be an appropriate tool to translate the conditions (i) and (ii) of theorem 1 into numerically tractable problems where the roles of $\mathcal{L}$ and $\mathcal{K}$ are clearly identified.

Theorem 2: [Quadratic stabilization] Let $\alpha$ be a given positive real numbers, and suppose there exist:

- two symmetric positive definite matrices $P_{c} \in \mathbb{R}^{2 \times 2}$ and $P_{o} \in \mathbb{R}^{(2 N+3) \times(2 N+3)}$,

- some gain matrices $\mathcal{L}$ and $\mathcal{K}$,

- two positive scalar $\gamma_{c}$ and $\gamma_{o}$

that satisfy the following relations where the dependancy of $\mathcal{A}$ on $\nu^{*}$ is ommitted:

$$
\left[\begin{array}{cc}
(A-B \mathcal{L})^{T} P_{c}+P_{c}(A-B \mathcal{L})+C_{c}^{T} C_{c}+2 \alpha P_{c} & P_{c} B \\
B^{T} P_{c} & -\gamma_{c}^{2}
\end{array}\right] \leq 0
$$

$$
\left[\begin{array}{cc}
(\mathcal{A}-\mathcal{K} \mathcal{C})^{T} P_{o}+P_{o}(\mathcal{A}-\mathcal{K} \mathcal{C})+C_{o}^{T} C_{o}+2 \alpha P_{o} & P_{o} B_{o} \\
B_{o}^{T} P_{o} & \left.-\gamma_{o}^{2} I\right] \leq 0
\end{array}\right.
$$

then the origin of (9) is globally exponentially stable with decay-rate $\alpha$ if the spatially periodic perturbation satisfies $2 \pi \gamma_{c} \gamma_{o} \gamma_{N}<1$, with $\gamma_{n}$ defined by (13).

Proof: Let $V_{c}\left(\chi_{c}\right)=\chi_{c}^{T} P_{c} \chi_{c}$ and $V_{o}\left(\chi_{o}\right)=\chi_{o}^{T} P_{o} \chi_{o}$, and differentiating these functions along the trajectories of (10) and (11) respectively, together with using the Schurr's complement of (14) and (15) yields:

$$
\begin{aligned}
& \frac{d}{d t}\left(\chi_{c}^{T} P_{c} \chi_{c}\right) \leq \gamma_{c}^{2} u_{c}^{T} u_{c}-\chi_{c}^{T} C_{c}^{T} C_{c} \chi_{c}-2 \alpha \chi_{c} P_{c} \chi_{c} \\
& \frac{d}{d t}\left(\chi_{o}^{T} P_{o} \chi_{o}\right) \leq \gamma_{o}^{2} u_{o}^{T} u_{o}-\chi_{o}^{T} C_{o}^{T} C_{o} \chi_{o}-2 \alpha \chi_{o} P_{o} \chi_{o}
\end{aligned}
$$

Assuming the small gain condition $2 \pi \gamma_{c} \gamma_{o} \gamma_{N}<1$ is fulfilled, there exists $\gamma_{c}^{2}<a<\frac{1}{4 \pi^{2} \gamma_{o}^{2} \gamma_{N}^{2}}$ so that : $\frac{d}{d t}\left(\chi_{c}^{T} P_{c} \chi_{c}+a \chi_{o}^{T} P_{o} \chi_{o}\right) \leq-2 \alpha\left(\chi_{c}^{T} P_{c} \chi_{c}+a \chi_{o}^{T} P_{o} \chi_{o}\right)$ 
which ends proving global exponential stability with decayrate $\alpha$, see for example [10] for further details.

\section{Controller gains tuning}

The recommended default PID settings for the motors yield closed loop eigenvalues that may be computed with the modeling (1). When omitting the cogging compensation feature of equation (8), our controller is an ordinary LTI controller with gain $\mathcal{L}$. We suggest to tune it to have eigenvalues with identical real part as with this PID. Without cogging compensation, we suggest to tune $\mathcal{L}$ to yield poles with real parts as negative as with the PID controller. If we rewrite $\mathcal{L}=\left(\begin{array}{ll}\omega^{2} & 2 \xi \omega-\mu\end{array}\right)$, it amounts to setting the poles of the transfer $H(s)$ :

$$
H(s)=\frac{s}{s^{2}+2 \xi \omega s+\omega^{2}} .
$$

$H(s)$ turns out ot be the transfer from $u_{c}$ to $\mathcal{C}_{c} \chi_{c}$ in (10). For $\mathcal{L}$ determined this way, we may also compute the smallest $\gamma_{c}$ fulfilling (14). It turns out to be the $H_{\infty}$ norm of the transfer $H(s-\alpha)$ given by:

$$
H(s-\alpha)=C_{c}((s-\alpha) I-A+B \mathcal{L})^{-1} B .
$$

This value is finite, and denoted by $\gamma_{c}^{*}$ provided the poles of $H(s)$ are located on the left of $-\alpha$ :

$$
\gamma_{c}^{*}=\|H(s-\alpha)\|_{\infty} .
$$

\section{Observer gains tuning}

For the previous controller gain $\mathcal{L}$, we propose to compute $\mathcal{K}$ in order to minimize the value of $\gamma_{o}$ for which the condition (15) is met. This problem is not straighforward, especially because $\mathcal{A}\left(\nu^{*}(t)\right)$ is a time-varying system. However, since the velocity along the reference trajectory is bounded, $\forall t, \underline{\nu} \leq \nu^{*}(t) \leq \bar{\nu}$, the system (11) is a polytopic system, and we are facing an optimization problem under Linear Matrix Inequalities constraints.

Theorem 3: The minimum value of $\gamma_{o}$ for which there exist $P=P^{T}>0$ and $\mathcal{K}$ such that (15) is fulfilled is $\gamma_{o}^{*}$, obtained by solving the following optimization problem:

$$
\left(\left(\gamma_{o}^{*}\right)^{2}, P^{*}, Q^{*}\right)=\min _{\gamma_{o}^{2}, \boldsymbol{P}, \boldsymbol{Q}} \gamma_{o}^{2}
$$

subject to:

$$
\begin{aligned}
& \exists \boldsymbol{P}=\boldsymbol{P}^{T}>0, \boldsymbol{P} \in \mathbb{R}^{(2 N+3) \times(2 N+3)} \\
& \exists \boldsymbol{Q} \in \mathbb{R}^{2 N+3} \\
& {\left[\begin{array}{cc}
\underline{\mathcal{A}}^{T} \boldsymbol{P}+\boldsymbol{P} \underline{\mathcal{A}}-\mathcal{C}^{T} \boldsymbol{Q}^{T}-\boldsymbol{Q} \mathcal{C}+C_{o}^{T} C_{o}+2 \alpha \boldsymbol{P} & \boldsymbol{P} B_{o} \\
B_{o}^{T} \boldsymbol{P} & -\boldsymbol{\gamma}_{\boldsymbol{o}}^{\mathbf{2}} I
\end{array}\right] \leq 0} \\
& {\left[\begin{array}{cc}
\overline{\mathcal{A}}^{T} \boldsymbol{P}+\boldsymbol{P} \overline{\mathcal{A}}-\mathcal{C}^{T} \boldsymbol{Q}^{T}-\boldsymbol{Q} \mathcal{C}+C_{o}^{T} C_{o}+2 \alpha \boldsymbol{P} & \boldsymbol{P} B_{o} \\
B_{o}^{T} \boldsymbol{P} & -\boldsymbol{\gamma}_{\boldsymbol{o}}^{\mathbf{2}} I
\end{array}\right] \leq 0}
\end{aligned}
$$

where $\underline{\mathcal{A}}=\mathcal{A}(\underline{\nu})$ and $\overline{\mathcal{A}}=\mathcal{A}(\bar{\nu})$ and the corresponding observer gain matrix $\mathcal{K}^{*}=\left(P^{*}\right)^{-1} Q^{*}$.

Proof: Since $\mathcal{A}\left(\nu^{*}\right)$ is a polytopic system, (15) only has to be enforced on the vertices $\underline{\mathcal{A}}$ and $\overline{\mathcal{A}}$ of $\mathcal{A}\left(\nu^{*}\right)$. See [10] for more details.

\begin{tabular}{|cc||c|c|ccc|}
\hline motor & controller & $\begin{array}{c}\text { Accuracy } \\
\text { in } \mu \mathbf{m}\end{array}$ & $\begin{array}{c}\lambda^{*} \\
\text { in } \mathrm{mm} / \mathrm{s}^{2}\end{array}$ & $\begin{array}{c}P_{1} \\
\text { in } \mathrm{mm}\end{array}$ & $P_{3}$ \\
\hline ironcore & $P I D$ & $\pm \mathbf{1 0}$ & - & - & - & - \\
ironcore & observer & $\pm \mathbf{0 . 5}$ & $\approx 5000$ & 24 & 16 & 12 \\
\hline ironless & PID & $\pm \mathbf{0 . 5}$ & - & - & - & - \\
ironless & observer & $\pm \mathbf{0 . 0 5}$ & $\approx 3500$ & 42 & 21 & - \\
\hline
\end{tabular}

TABLE I

EXPERIMENTAL SETUP AND RESULTS. COMPARISON OF THE PROPOSED METHOD WITH A PID CONTROLLER.

\section{E. Conclusion}

The observer-based controller made up of (6) and (8) is globally exponentially stable with decay-rate to zero $\alpha$ for perturbations (2) fulfilling the relation:

$$
\gamma_{N}<\gamma_{N}^{*}=\frac{1}{2 \pi\|H(s-\alpha)\|_{\infty} \gamma_{o}^{*}},
$$

with $\gamma_{N}$ given by (13), $\gamma_{o}^{*}$ in the theorem 3 and $H(s)$ in subsection IV-C. This is the largest perturbation for which the stability of the method is proved, and may be regarded as a robustness margin. Computing the numerical value of $\gamma_{N}^{*}$ allows to estimate the largest perturbations this controller may withstand by computing the maximum admissible value $\lambda^{*}$ of the $\lambda_{n}$. This shows the proposed design can cope with perturbations hardly conceivable from a physical point of view, as illustrated in table I.

\section{EXPERIMENTAL RESULTS}

We consider two commercial off-the-shelve PM linear motors, both an ironcore and an ironless motor. They are intended to carry loads, for point to point motion, with respective reference speed trajectories given on figure 2. In order to determine the spatial periods $P_{n}$ of the equation (2), we drive each motor with its recommended default PID controller and perform an FFT transform of the recorded tracking error. These results are plotted in blue dotted line on the figures 4 and 3 .

At this point, it is noteworthy that the spectral content of the cogging forces is much more intricate in the case of the ironcore motor than with the ironless one. The latter clearly features two spatial periods located at $42 \mathrm{~mm}$ and $21 \mathrm{~mm}$, while the ironcore motor spectrum is much more chaotic. Nevertheless, the spatial periods $24 \mathrm{~mm}, 16 \mathrm{~mm}$ and $12 \mathrm{~mm}$ are used to tune the observer. In other words, the assumption about the spatially periodic nature of the cogging forces for the ironcore motor is not as well fulfilled as for the ironless one.

We still implement our observer-based controller for both the motors and get the results presented on figure 4 and table I. The cogging forces are completely filtered out (see the figure 3 for a quantitative illustration), after a short transient for both motors. As expected, this design is efficient during 


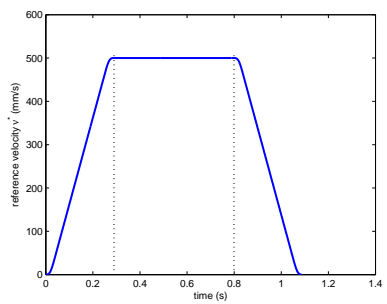

(a) Ironcore motor.

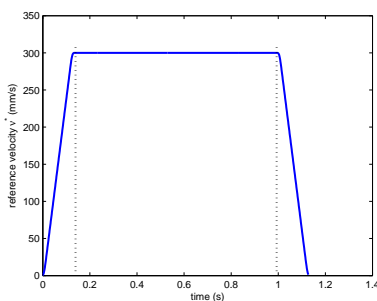

(b) Ironless motor.
Fig. 2. Reference velocity profiles in $\mathrm{mm} / \mathrm{s}$.

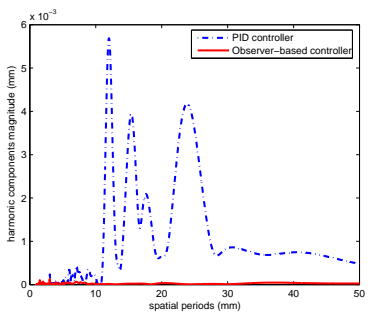

(a) Ironcore motor.

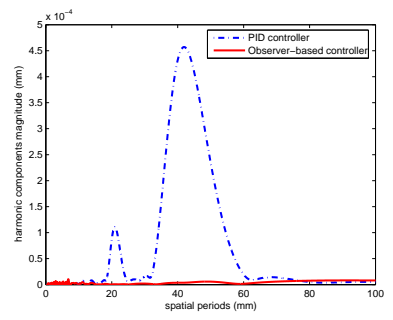

(b) Ironless motor.
Fig. 3. FFT of the tracking error wrt. spatial perdiods in $\mathrm{mm}$.

the constant velocity phases as well as the during acceleration and deceleration phases.

Let's also mention that $\lambda^{*}$ (the largest admissible $\lambda_{n}$ for which stability is guaranteed) is given in table I. The nominal acceleration for the reference speed profiles of figure 2 are $4000 \mathrm{~mm} / \mathrm{s}^{2}$ (resp. $2500 \mathrm{~mm} / \mathrm{s}^{2}$ ) for the ironcore (resp. ironless) motor. This means that, if the cogging forces acting on these motors ever had the same magnitude as the nominal thrust, the stability of this controller would not be jeopardized. In view of the efforts made by motors manufacturers to reduce them, cogging forces turn out to be perturbations, whose magnitude actually do not reach such values. We believe our approach allows to cope with most practical use conditions.

In the end, as illustrated in table I, for the ironless motor, the tracking error is 10 times smaller than with a PID, and 20 times smaller for the ironcore motor. One may also note that the ironcore motor driven by the observer-based controller reaches the intrinsic performances of the ironless motor with a PID. In other words, our solution makes ironcore motor perform as well as ironless motors driven by a casual control scheme. On the top of that, ironless motors have their performances clearly improved.

\section{CONCLUSION}

A new controller scheme dedicated to cogging forces cancellation has been proposed. This approach only relies on position measurements, is based on physical modeling of these perturbations. It estimates these perturbations online to then get rid of them. As illustrated through the proposed experimental data, the desired trajectory can be of any kind, and, after a short transient, the motor reaches the reference trajectory. This cogging dedicated controller

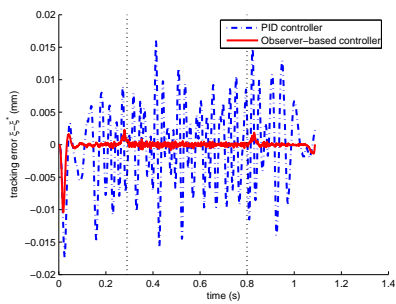

(a) Ironcore motor.

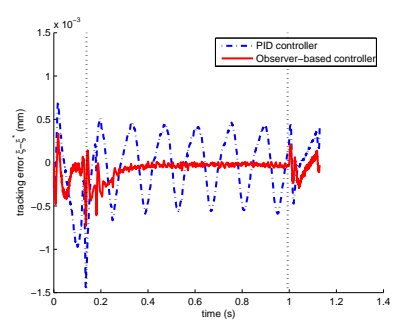

(b) Ironless motor.
Fig. 4. Experimental tracking error in $\mu m$, with a PID controller (- - ) and the observer-based controller (-).

significantly removes unsatisfactory oscillations around the reference trajectory, which makes the proposed controller scheme definitely suited for high accuracy applications, such as in the semiconductor industry. Ironcore motors may thus achieve accuracy usually met with ironless motors, themselves reaching upstream performances.

\section{REFERENCES}

[1] J. Wang, D. Howe, and G. Jewel, "Fringing in tubular permanentmagnet machines: Part I, magnetic field distribution, flux linkage, and thrust force," IEEE Transactions on Magnetics, vol. 39, no. 6, September 2003.

[2] — , "Fringing in tubular permanent-magnet machines: Part II, cogging force and its minimization," IEEE Transactions on Magnetics, vol. 39, no. 6, November 2003.

[3] X. Wang, Y. Yang, and D. Fu, "Study of cogging torque in surfacemounted permanent magnet motors with energy method," Journal of magnetism and magnetic materials, vol. 267, 2003.

[4] M. Islam, S. Mir, and T. Sebastian, "Issues in reducing the cogging torque of mass-produced permanent-magnet brushless dc motor," IEEE Transactions on Industry Applications, vol. 40, no. 3, May/June 2004.

[5] G. Otten, J. de Vries, J. van Amerongen, M. Rankers, and W. Gall, "Linear motor motion control using a learning feedforward controller," IEEE/ASME transactions on mechatronics, vol. 2, no. 3, September 1997.

[6] H.-S. Ahn, Y. Chen, and H. Dou, "State-periodic adaptive compensation of cogging and coulomb friction in permanent-magnet linear motors," IEEE Transactions on Magnetics, vol. 41, no. 1, January 2005.

[7] K. Tan, S. Huang, and T. Lee, "Robust adaptive numerical compensation for friction anf force ripple in permanent-magnet linear motor,' IEEE Transactions on Magnetics, vol. 38, no. 1, January 2002.

[8] C. Canudas de Wit and L. Praly, "Adaptive eccentricity compensation," IEEE Transactions on Control Systems Technology, vol. 8, no. 5, September 2000.

[9] J. Malaizé and J. Lévine, "Active estimation of the initial phase for brushless synchronous motors," in Proceedings of the 9th IEEE International Worshop on Advanced Motion Control, Istanbul, Turkey, 2006.

[10] S. Boyd, L. El Ghaoui, E. Feron, and V. Balakrishnan, Linear Matrix Inequalities in System and Control Theory. SIAM, 1994. 